\title{
Y-type partial duplication of a vaginal ectopic ureter with ipsilateral hypoplastic pelvic kidney and bicornuate uterus
}

\author{
Shushang $\underline{C h e n}^{1}$, PhD, Lingfeng $\underline{Z h u^{1}}$, MD, Shunliang $\underline{Y a n g}^{1}, \mathrm{MD}$, Jianming $\underline{\operatorname{Tan}}^{1}$, PhD
}

\begin{abstract}
We present a case of vaginal ectopic ureter with ipsilateral partial duplication of the upper ureter (Y-type ureter), ipsilateral hypoplastic pelvic kidney and bicornuate uterus in a 20-year-old woman, who presented with mild urinary incontinence since infancy. Ultrasonography, computed tomography and intravenous pyelography examination showed a left kidney with no evidence of a right kidney. Cystourethroscopy showed absence of the right hemitrigone. Magnetic resonance (MR) urography demonstrated the presence of a bicornuate uterus, an ectopic dysplastic right kidney in the pelvic cavity, and a right ureter that terminated in the fornix vaginae. The patient underwent right nephroureterectomy, and urinary continence was restored completely. Although congenital malformations of the urinary tract are frequently associated with genital tract abnormalities, this is, to the best of our knowledge, the first report of the coexistence of these anomalies in an individual. Our report also highlights the importance of MR urography in the diagnosis of such rare and complex anomalies.
\end{abstract}

Keywords: bicornuate uterus, duplication, ectopic kidney, vaginal ectopic ureter

\section{INTRODUCTION}

Ectopic ureter with partial ureteral duplication is extremely rare. We report the case of a 20 -year-old woman who was diagnosed with vaginal ectopic ureter, ipsilateral partial duplication of the upper ureter (Y-type ureter), ipsilateral hypoplastic pelvic kidney and bicornuate uterus. Although congenital malformations of the urinary tract are frequently associated with genital tract abnormalities, ${ }^{(1,2)}$ this is, to the best of our knowledge, the first report of the concurrent coexistence of all of these anomalies in an individual.

\section{CASE REPORT}

A 20-year-old woman presented with mild, constant urinary dribbling since infancy. She had normal menses and voiding habits, with no symptoms of dysuria, urinary urgency or urinary frequency. No history suggestive of stress incontinence was noted. Due to the constant dribbling of urine, the patient had to use pads daily. Since childhood, the patient had undergone a series of noninvasive and invasive diagnostic procedures including ultrasonography and excretory urography, but the cause of the urinary dribbling could not be ascertained.

The patient was admitted to the Department of Urology in January 2011. On physical examination, an opening that measured approximately $0.8 \mathrm{~cm}$ in diameter, with slight leakage of light yellow urine, was observed at the external vaginal orifice. Abdominal ultrasonography and computed tomography examination showed a compensatory hypertrophied left kidney and no obvious evidence of a right kidney. Intravenous pyelography (IVP) showed normal excretion from a single normal left renal pelvis and absence of the right collecting system.
Cystourethroscopy revealed that the right hemitrigone was absent and that the left ureteral orifice was normally situated. No ectopic ureteral opening was observed in the bladder and urethra. Vaginoscopy was not performed because the patient was a maiden and did not consent to the procedure. Magnetic resonance $(M R)$ imaging and $M R$ urography was then conducted; these imaging studies revealed the presence of an ectopic dysplastic right kidney in the pelvic cavity, measuring $2 \mathrm{~cm} \times 2.5 \mathrm{~cm}$. The right ureter appeared to pass behind the bladder and terminate in the fornix vaginae. A bicornuate uterus was also detected, and the bilateral adnexa were observed to be normal (Fig. 1).

The patient subsequently underwent open right nephroureterectomy. Intraoperatively, we found that the right ureter had partial duplication of the upper part, forming a Y-type ureter, which entered the right lateral wall of the vagina. We also found a hypoplastic kidney (Fig. 2), measuring $3.5 \mathrm{~cm} \times 2 \mathrm{~cm} \times 2 \mathrm{~cm}$, beneath the right common iliac vessels. The ectopic kidney and ureter were completely resected. Post operation, the patient's urinary continence was restored completely and follow-up was unremarkable.

\section{DISCUSSION}

A normal site of ureter insertion is necessary for the normal development of the ipsilateral kidney. During gestation, abnormal development of the mesonephric (wolffian) and paramesonephric (mullerian) ductal systems results in ureteric ectopia, which subsequently leads to the maldevelopment, absence, or ectopia of the ipsilateral kidney.(1) Ectopic ureters may arise from the upper pole of a completely duplicated 

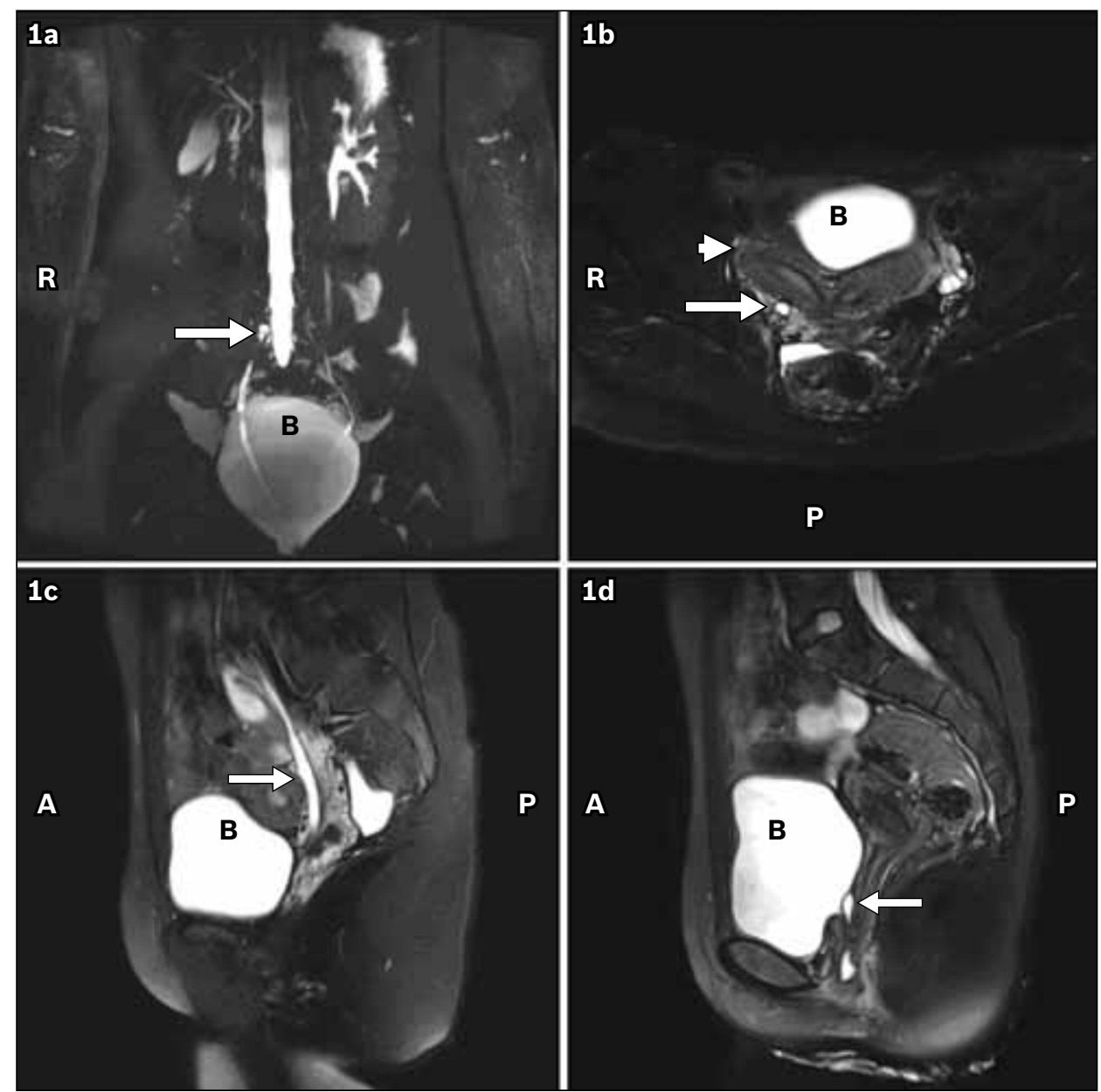

Fig. 1 (a) MR urography shows the absence of a kidney in the right renal fossa and a dysplastic ectopic right kidney (arrow) in the pelvic cavity, with the right ectopic ureter passing behind the bladder. (b) T2-W MR image shows the bicornuate uterus (arrowhead) and the right ectopic ureter (arrow) passing behind the right uterus. (c) T2-W MR image shows the right ectopic ureter (arrow) passing behind the right uterus and bladder. (d) T2-W MR image shows the right ectopic ureter terminating in the fornix of the vagina (arrow). A: anterior; B: bladder; P: posterior; R: right

system (about $80 \%$ ) or have a single draining system (about 20\%). ${ }^{(3,4)}$ Due to the close embryological interaction between the uropoietic and genital systems, congenital genital tract abnormalities are frequently associated with malformations in the urinary tract and vice versa. ${ }^{(1)}$ Bicornuate uteruses associated with vaginal ectopic ureters have been reported in cases with an ipsilateral, completely duplicated system, ${ }^{(1)}$ single-draining system, ${ }^{(2)}$ and inverted-Y ureteral duplication. ${ }^{(5)}$ The present case of bicornuate uterus and vaginal ectopic ureter with ipsilateral, partially duplicated Y-type ureter and hypoplastic pelvic kidney is unique and interesting.

Morphological data about the course of the ureter and the location of the ectopic insertion are of utmost importance for diagnosis and in planning treatment strategy. Ultrasonography is a noninvasive and useful imaging tool that is typically used in the initial investigation. It is considered the investigation of choice for the diagnosis of anomalies of the urinary tract. However, detection of small ectopic hypoplastic renal tissue and identification of the insertion site of an ectopic ureter may be difficult with ultrasonography. In our case, the patient was misdiagnosed as having solitary kidney by repeated ultrasonography. Therefore, women with solitary kidneys should also receive thorough ultrasonic evaluation of the pelvis to detect any ectopic renal tissue.(1) Although IVP, voiding cystourethrogram and cystourethroscopy are important complementary imaging techniques for the assessment of ectopic ureters, these techniques often yield incomplete diagnostic information. As seen in our case, imaging of the ectopic ureter was a diagnostic challenge due to the poor function of the ipsilateral kidney. IVP showed normal excretion from the normal left kidney with a single renal pelvis and absence of the right renal collecting system. On cystourethroscopy, the right hemitrigone was absent. These findings may lead to a misdiagnosis of congenital solitary kidney. Nevertheless, cystourethroscopy may help to rule out other causes of urinary incontinence such as pathological changes in the bladder neck. Like IVP, a radionuclide scan may not detect a dysplastic ectopic kidney with severely reduced function because of its dependence on the renal handling of radioisotopes. ${ }^{(2)}$ CT imaging can provide 3D anatomical data for identifying urinary abnormity, ${ }^{(4,5)}$ but may fail to show the entire course of the ectopic ureter if the ipsilateral kidney is too hypoplastic to 


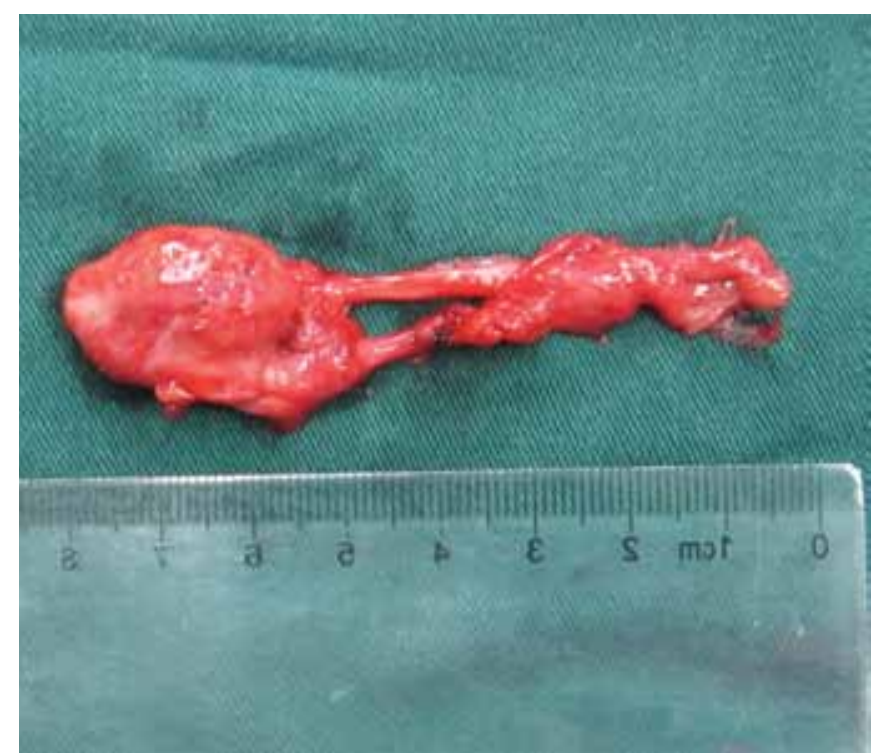

Fig. 2 Photograph shows the excised dysplastic ectopic right kidney with Y-type ureter.

handle the contrast material. Furthermore, the high radiation exposure of $\mathrm{CT}$ imaging carries potential risks to children.

MR urography has been demonstrated to be an excellent investigation modality in cases of complex genitourinary abnormality. ${ }^{(2)}$ It is able to accurately depict dysplastic ectopic renal tissue, the entire course of an ectopic ureter, and the ectopic ureter's site of insertion. ${ }^{(5)}$ Furthermore, there is no risk of ionising radiating exposure with MR imaging. In our case, preoperative MR imaging and MR urography successfully revealed the vaginal ectopic ureter with ipsilateral, hypoplastic pelvic kidney and bicornuate uterus, but failed to detect the partial duplication of the upper ureter, which was only diagnosed intraoperatively. Nevertheless, MR imaging and MR urography are still valuable investigations of choice as these investigations are able to provide accurate anatomical and functional information in cases of complex congenital abnormality.

Treatment of the ectopic ureter depends on the functionality of the ipsilateral and contralateral kidney. If good functional renal parenchymal tissue is present, ureteral reimplantation is recommended; if the ipsilateral kidney is hypoplastic with very poor functionality and the contralateral kidney is normal, nephroureterectomy may be an appropriate option.

\section{REFERENCES}

1. Dwyer PL, Rosamilia A. Congenital urogenital anomalies that are associated with the persistence of Gartner's duct: A review. Am J Obstet Gynecol 2006; 195:354-9.

2. Jackson L, Murphy FL, Hiorns MP, Duffy P. The role of dynamic magnetic resonance urography in complex neonatal hydrometrocolpos. Pediatr Surg Int 2008; 24:625-7.

3. Kumar S, Bera MK, Bera KP, Vijay MK, Kundu AK. Laparoscopic ureteric reimplantation of a single-system ectopic ureter in a girl: A rarity. J Minim Access Surg 2010; 6:80-2.

4. Croitoru S, Gross M, Barmeir E. Duplicated ectopic ureter with vaginal insertion: 3D CT urography with i.v. and percutaneous contrast administration. AJR Am J Roentgenol 2007; 189:W272-4.

5. Liu L, Yang J, Zhu L, et al. Crossed-fused renal ectopia associated with inverted-Y ureteral duplication, ectopic ureter, and bicornuate uteruses. Urology 2010; 75:1175-7. 